\title{
Cholera Toxin: Molecular Mechanisms and Drug Design
}

Ute Krengel, Department of Chemistry, University of Oslo

Cholera is an ancient diarrheal disease that has its origins in the Ganges delta, and from there conquered the rest of the world. Epidemics occur particularly where disaster strikes, most recently in Yemen. Individuals with blood group $\mathrm{O}$ are at increased risk of severe symptoms but why? We have recently contributed to solving this mystery with several high-resolution crystal structures in complex with blood group determinants ${ }^{1}$. Together with quantitative binding data from SPR analyses, they suggest a molecular mechanism for this phenomenon. They further suggest why breast-fed infants may be protected from the disease. For those individuals that are not so fortunate, there are currently three options: vaccinate, take antibiotics or count on your immune system, with a helping hand from oral rehydration solution (or intravenous fluids). We hope to soon add a fourth: cholera prophylactics ${ }^{2}$.

${ }^{1}$ Heggelund et al. (2016) High-resolution crystal structures elucidate the molecular basis of cholera blood group dependence. PLoS Pathog. 12(4): e1005567.

${ }^{2}$ Heggelund et al. (2017) Towards new cholera prophylactics and treatment: Crystal structures of bacterial enterotoxins in complex with GM1 mimics. Sci Rep. 7(1): 2326. 